\title{
数值模型驱动的传动系统故障个性化诊断原理
}

\author{
向家伟 \\ (温州大学机电工程学院 温州 325035)
}

\begin{abstract}
摘要: 如何获得机械传动系统大量反映实际运行状态的故障样本, 是制约人工智能诊断模型走向工程应用的瓶颈。基于个体 差异的精准诊断需求, 提出机械传动系统个性化故障诊断基本原理, 通过建立机械传动系统数值模型, 进行仿真分析, 获得 故障样本, 解决故障诊断过程中故障特征信息缺乏的短板, 从而激活人工智能沴断方法。以轴承、齿轮传动、转子系统等机 械传动系统为例, 构建完好结构有限元模型, 开展模型修正, 获得具有一定精度的仿真模型。预定义多类故障并添加至具有 一定精度的有限元模型, 计算生成故障样本集, 作为人工智能诊断模型的训练样本, 用于待诊断测试样本分类。任意选取的 支持向量机、极限学习机、卷积神经网络等人工智能诊断模型故障分类实验结果表明: 所提出机械传动系统故障诊断的个性 化诊断原理, 具有较强的普适性与可拓展性。
\end{abstract}

关键词：机械传动系统；故障；个性化诊断；人工智能诊断模型；分类

中图分类号: $\mathrm{TH} 322$

\section{Numerical Model Driving Personalized Diagnosis Principle for Fault Detection in Mechanical Transmission Systems}

\author{
XIANG Jiawei \\ (College of Mechanical \& Electrical Engineering, Wenzhou University, Wenzhou 325035)
}

\begin{abstract}
How to obtain a large number of fault samples from mechanical transmission systems under the actual running state is a bottleneck for the engineering application using intelligent diagnosis methods. To meet the requirement of precision diagnosis for individual differences, the basic principle of personalized diagnosis for mechanical faults is proposed. Through the construction of numerical simulation model of mechanical transmission systems, simulations are performed to obtain fault samples. The bottleneck problem of lacking fault feature information in the diagnostic procedures will be resolved to activate the artificial intelligent (AI) diagnosis methods. Taking the bearing, gear transmission, rotor-bearing system for examples, the finite element method (FEM) models of intact structures are firstly constructed to obtain simulation model with a certain precision using model updating techniques. Secondly, predefined several faults and further inserted into the high fidelity FEM model to calculate the fault samples, which severed as training samples of AI diagnostic models to classify testing samples (faults to be diagnosed). Finally, the experimental investigations using the arbitrary selection of support vector machine (SVM), extreme learning machine (ELM) and convolutional neural network $(\mathrm{CNN})$ show that the principle of personalized diagnosis for mechanical faults has strong universality and expansibility.
\end{abstract}

Key words: mechanical transmission systems; faults; personalized diagnosis; artificial intelligent models; classification

\section{0 前言}

车辆、风力发电机组、化工过程机械、工程机 械等装备的机械传动系统, 长期在重载、疲劳、腐

* 国家自然科学基金资助项目(U1909217, U1709208)。20200904 收到初 稿, 20210218 收到修改稿
蚀、高温等复杂恶劣的工况下运行，系统中的核心 零部件不可避免地产生不同程度的损伤, 譬如：主 轴、齿轮、轴承经常出现磨损、破损、断齿、齿根 裂纹、剥落、胶合、擦伤等损伤现象。机械传动系 统一旦出现事故，将带来巨大的经济损失和人员伤 亡。因此，国内外在机械工程领域开展了大量针对 机械传动系统主轴、齿轮、轴承的损伤故障诊断研 究 $^{[1]}$, 提出了诸多人工智能诊断模型, 如: 贝叶斯 
理论、模糊集合理论、D-S 证据推理、遗传算法、 聚类分析、支持向量机 (Sapport vector machine, SVM)、极限学习机 (Extreme learning machine, ELM)、卷积神经网络(Convolutional neural network, $\mathrm{CNN}$ )等 ${ }^{[2]}$, 这为机械传动系统故障诊断提供了基本 工具, 已成为机械故障诊断领域研究的一个热点与 有前途的研究方向 ${ }^{[3-5]}$ 。人工智能诊断模型的应用可 以摆脱故障诊断过分依赖于专业技术人员和诊断专 家的问题, 在线实现高效、可靠的诊断, 其作用和 效益日趋显著, 覆盖面越来越宽, 并为诸多研究者 所充分肯定 ${ }^{[6-8]}$ 。然而, 实际运行设备损伤故障特征 信息微弱, 往往被机械传动系统的强噪声所淹没, 并且试验室数据与实际设备运行数据不属于同一母 体, 具有 “个性化” 特征, 只能用于研究故障现象 的一般原理和发展规律, 而故障监测诊断需要的是 针对某台机械设备的个体数据。即使同一型号的机 械设备, 由于装配、安装及运行条件不同, 其工况 状态边界是有差别的, 必须从实际设备运行状态出 发, 获取大量能反映实际运行条件的不同类型故障 样本 ${ }^{[9]}$ 。如何获得机械传动系统大量能反映实际运 行状态的故障特征信息, 是制约这些优秀的人工智 能诊断模型走向工程应用的瓶颈。若能采用数值模 拟/仿真分析手段, 建立机械传动系统仿真分析模 型, 进行高性能仿真分析, 获得大量反映实际运行 条件的不同类型单、多故障样本, 解决现有人工智 能诊断模型缺乏故障样本的难题, 探索特定工作状 态下机械传动系统故障诊断新途径, 必将有效地提 高重载、疲劳、腐蚀、高温等复杂恶劣的工况下运 行的机械传动系统的服役性能, 具有重要的学术意 义和应用价值。

个性化诊断 ${ }^{[10]}$ 是近年来随着分子生物学发展 起来的一种基于个体差异的精确诊断技术, 这无疑 为借鉴医学诊断技术而发展起来的机械故障诊断理 论和方法提供新思路。随着机械系统动力学的发展, 有可能将机械系统动力学数值模型应用于具体运行 工况下机械装备故障样本模拟, 为人工智能诊断提 供足够多的故障样本, 实现基于数值模型的机械传 动系统个性化诊断。

近年来, 为开展故障机理研究, 国内外学者在 损伤结构动力学模型方面进行了广泛的探索, 如: 文献[11]采用传递矩阵法, 建立了含故障的曲柄活 塞机构动力学模型并分析其大端轴承的故障机理; 文献[12]考虑行星架损伤, 采用集中质量法建立行 星齿轮传动系统非线性动力学模型, 通过仿真分析,
得出了系统非线性动态响应和损伤程度之间的规 律; 文献[13]推导了行星轮系正常、裂纹及剥落三 种情况下的时变啮合刚度算法, 在考虑振动传递路 径时变效应的影响的基础上, 建立了相应的动力学 模型, 求解出行星轮系正常、裂纹及剥落时的动态 响应及频谱特性; 文献[14]对基于旋转机械损伤动 力学模型的故障预测技术进行了综述。然而, 实际 机械结构系统往往传递环节众多, 为研究其关键零 件损伤机理, 一些研究者进行了开创性探索, 提出 了一些损伤动力学模型, 如: 考虑预啮合刚度变化 和间隙非线性的单级齿轮传动系统非线性动力学有 限元模型 ${ }^{[15]}$; 损伤引起的时变载荷作用下圆柱滚子 轴承系统有限元模型 ${ }^{[16]}$ 等。以上研究为机械传动系 统故障模拟提供了可用的数值模型。然而, 仅依赖 数值模型本身, 不能保证其与实际物理系统之间完 全匹配, 为消除机械传动系统在实际运行状态下不 确定性的影响(如边界条件、阻尼、零部件配合状况 等), 需要利用少量物理试验, 通过测试与数值模型 仿真得到的动态响应匹配分析, 对理论模型中敏感 的参数进行修正 ${ }^{[17]}$ 。

近年来,在国家自然科学基金和浙江省杰出青 年基金的资助下，作者团队已开展数值模型驱动 的机械传动系统故障个性化诊断具体方法研究, 通过建立轴承-转子系统 ${ }^{[18]}$ 、齿轮传动系统 ${ }^{[19]}$ 、轴 承 ${ }^{[20]}$ 等机械传动系统关键零部件的有限元数值模 型, 仿真获得大量故障样本, 作为人工智能模型 的训练样本, 有望从根本上解决智能诊断过程中 故障样本缺乏的短板, 激活人工智能模型, 搭建 人工智能诊断模型走向具有 “个性化” 特征的机 械装备应用的桥梁, 形成数值模型驱动的机械传 动系统故障个性化诊断基本原理。

\section{1 数值模型驱动的机械传动系统故障 个性化诊断基本原理}

图 1 所示为数值模型驱动的机械传动系统故障 个性化诊断基本原理。

(1) 数值模型建立及修正。

利用有限元法、传递矩阵法、集中质量法等构 建机械传动系统的初始数值模型。众所周知, 振动 响应信号是机械设备动力学特性的直接反映, 蕴含 了刚度、阻尼、载荷等重要参数。为使数值模型与 实际物理系统振动响应信号匹配, 消除机械传动系 
统在实际运行状态下边界条件、阻尼、零部件配合 状况等影响, 可利用距离(欧式距离、明可夫斯基距 离、海明距离)或相似度(余弦相似度、皮尔森相关 系数、对数似然相似度) 等数据相似性度量标准进行 匹配分析。本文采用余弦相似度 $\cos \theta^{[21]}$

$$
\cos \theta=\frac{\boldsymbol{V}_{\mathrm{p}} \cdot \boldsymbol{V}_{\mathrm{s}}}{\left\|\boldsymbol{V}_{\mathrm{p}}\right\| \times\left\|\boldsymbol{V}_{\mathrm{s}}\right\|}=\frac{\sum_{i=1}^{N} V_{\mathrm{p}}^{i} \times V_{\mathrm{s}}^{i}}{\sqrt{\sum_{i=1}^{N}\left(V_{\mathrm{p}}^{i}\right)^{2}} \times \sqrt{\sum_{i=1}^{N}\left(V_{\mathrm{s}}^{i}\right)^{2}}}
$$

式中, $\boldsymbol{V}_{\mathrm{p}}$ 与 $\boldsymbol{V}_{\mathrm{s}}$ 分别为物理系统测试与数值模型仿真 得到的长度为 $N$ 的振动响应。

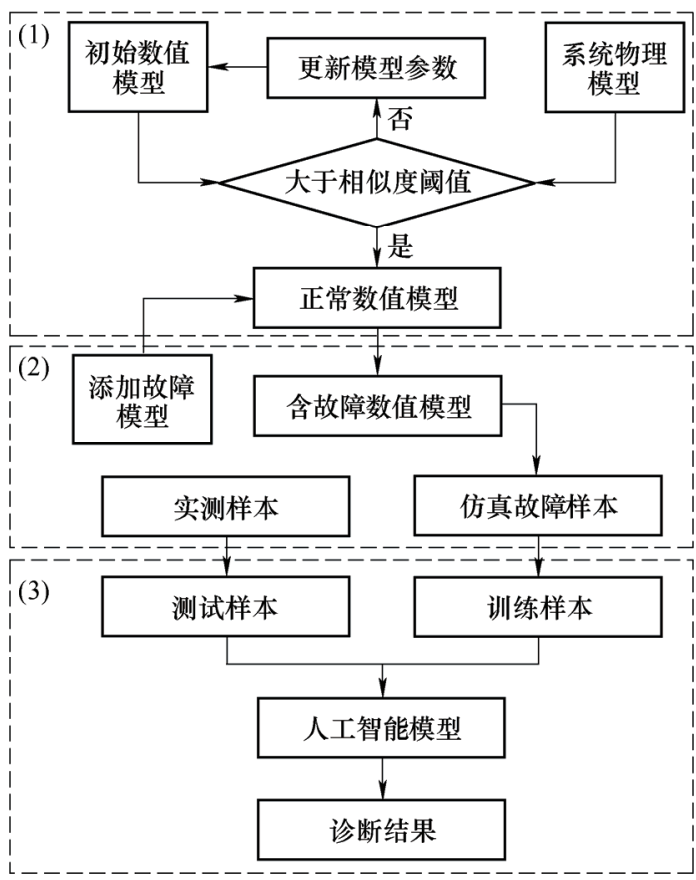

图 1 机械传动系统故障个性化诊断流程图

一般而言，当二者相似度超过预设阈值 $0.6^{[21]}$ 时, 可认为修正的数值模型有效, 否则更新数值模 型参数, 迭代求解, 直至达到预设阈值要求, 最终 建立机械传动系统正常状态下的具有一定精度的数 值模型。值得指出的是: 预设阈值越大, 模型修正 效果越好，但模型修正过程计算量越大。

(2) 获取训练样本。

预定义机械传动系统可能出现的故障模式, 构 建故障几何模型或数学模型并添加至正常状态下的 具有一定精度的数值模型, 获得含故障机械传动系 统数值模型, 进行振动响应模拟计算, 得到能反映 实际机械传动系统故障状态的仿真信号。根据原始 信号消噪及人工智能诊断模型对输入数据的要求, 将每个仿真得到的原始信号做基于统计分析或信号 处理方法的预处理, 构建仿真故障样本, 作为人工 智能模型的训练样本。
(3) 识别故障类型。

定期或在线对机械传动系统进行振动测试, 获 得实测原始信号, 同样根据原始信号消噪及人工智 能诊断模型对输入数据的要求, 将每个实测原始信 号做基于统计或信号处理方法的预处理，构建待诊 断的实测样本, 作为人工智能模型的测试样本。将 测试样本与训练样本一起输入至人工智能模型, 最 终识别出故障类型, 从而实现数值模型驱动的机械 传动系统故障个性化诊断。

为验证数值模型驱动的机械传动系统故障个性 化诊断原理的有效性, 本文给出了机械传动系统典 型零部件, 如: 轴承、齿轮、轴承——转子系统个 性化诊断的具体方法。

\section{2 零部件故障个性化诊断具体方法}

\section{1 有限元模拟驱动 SVM 的轴承故障个性化诊断 方法}

针对轴承故障类型识别问题, 提出一种有限元 模拟驱动 SVM 的轴承个性化诊断方法。预处理采 用时域统计量, 通过计算原始信号在时域上的 16 个指标(见表 1: $x$ 是一个数据序列, $N$ 是数据点个 数), 构建训练样本和测试样本。

表 1 时域指标及其计算公式

\begin{tabular}{cc}
\hline 时域指标 & $x_{\mathrm{m}}=\sum_{i=1}^{N} x_{i} / N$ \\
\hline 均值 $x_{\mathrm{m}}$ & $x_{\mathrm{ava}}=\sum_{i=1}^{N}|x(i)| / N$ \\
绝对平均值 $x_{\mathrm{ava}}$ & $x_{\mathrm{std}}=\sqrt{\sum_{i=1}^{N}\left(x(i)-x_{\mathrm{m}}\right)^{2} / N}$ \\
标准差 $x_{\mathrm{std}}$ & $x_{\mathrm{sra}}=\left(\sum_{i=1}^{N} \sqrt{|x(i)|} / N\right)^{2}$ \\
均方根幅值 $x_{\mathrm{sra}}$ & $x_{\mathrm{var}}=\sum_{i=1}^{N}\left(x_{i}-\mathrm{x}_{\mathrm{m}}\right)^{2} / N$ \\
方差 $x_{\mathrm{var}}$ & $x_{\mathrm{ske}}=\sum_{i=1}^{N}\left(x(i)-x_{\mathrm{m}}\right)^{3} / N$ \\
斜度 $x_{\mathrm{ske}}$ & $x_{\mathrm{p}}=\max |x(i)|$ \\
峰值 $x_{\mathrm{p}}$ & $x_{\mathrm{kur}}=x_{\mathrm{std}}^{4} \sum_{i=1}^{N}\left(x(i)-x_{\mathrm{m}}\right)^{4} / N$ \\
峭度 $x_{\mathrm{kur}}$ & $x_{\max }=\max (x(i))$ \\
最大值 $x_{\max }$ & $S F=x_{\mathrm{rms}} /\left(\sum_{i=1}^{N}|x(i)| / N\right)$ \\
波形指数 $S F$ & $x_{\min }=\min (x(i))$ \\
最小值 $x_{\min }$ & $I F=x_{\mathrm{p}} /\left(\sum_{i=1}^{N}|x(i)| / N\right)$ \\
脉冲因子 $I F$ & $x_{\mathrm{pp}}=\max (x(i))-\min (x(i))$ \\
峰峰值 $x_{\mathrm{pp}}$ & $P F=x_{\mathrm{p}} / x_{\mathrm{rms}}$ \\
峰值因子 $P F$ & $x_{\mathrm{rms}}=\sqrt{\sum_{i=1}^{N}\left(x_{i}\right)^{2} / N}$ \\
均方根值 $x_{\mathrm{rms}}$ & $x_{\max } /\left(\sum_{i=1}^{N} \sqrt{|x(i)| / N)^{2}}\right.$ \\
裕度指标 $C I$ &
\end{tabular}

图 2 所示为轴承故障个性化诊断方法流程。 
(1) 建立正常轴承的初始有限元模型, 计算获 得仿真振动加速度响应信号。

(2) 构建测试系统, 在轴承座上通过加速度传 感器获得轴承振动加速度实测信号。

(3) 将余弦相似度 $\cos \theta$ 作为仿真与实测振动信 号相似性度量标准, 迭代调整轴承有限元模型未定 参数, 当 $\cos \theta>0.6$ 时, 停止迭代并获得未定参数值, 完成轴承具有一定精度的有限元模型。

(4) 预定义轴承可能出现的故障模式, 在具有 一定精度的有限元模型中嵌入故障几何模型, 获得 含故障轴承有限元模型, 进行振动响应有限元模拟 计算, 得到轴承故障状态的仿真信号。

(5) 将故障仿真信号作归一化处理, 在时域上 分割成若干长度相同的子信号, 并根据表 1 公式, 计算每个子信号的 16 个时域指标, 完成仿真故障样 本构建, 作为 SVM 模型的训练样本。

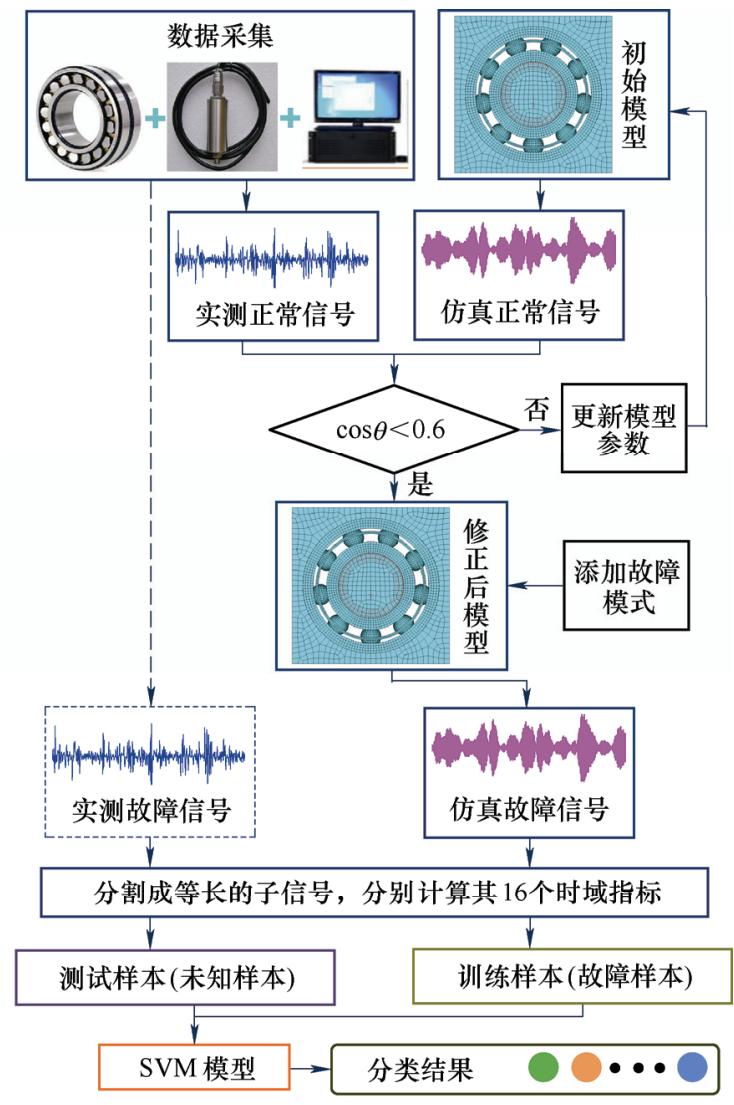

图 2 轴承故障个性化诊断方法流程图

(6) 采集运行中轴承座上振动信号, 获得实测 故障信号, 将实测故障信号作与故障仿真信号同 样的归一化处理、分割、计算时域指标, 完成实 测故障样本构建, 作为 SVM 模型的测试样本。将 测试样本与训练样本一起输入至 SVM 模型, 最终 识别出故障类型, 从而实现轴承故障的个性化 诊断。

\section{2 有限元模拟驱动 ELM 的齿轮故障个性化诊断 方法}

为解决齿轮故障精准诊断问题, 提出一种有限 元模拟驱动 ELM 的个性化诊断方法, 该方法融合 了有限元仿真技术模拟任何状态下齿轮故障的能力 和 ELM 能最小化训练误差和权值范围的优点。相 比轴承故障诊断, 由于传感器采集信号传递路径复 杂, 振动信号噪声干扰更强烈, 因此, 本文给出如 图 3 所示的齿轮故障个性化诊断方法基本流程。

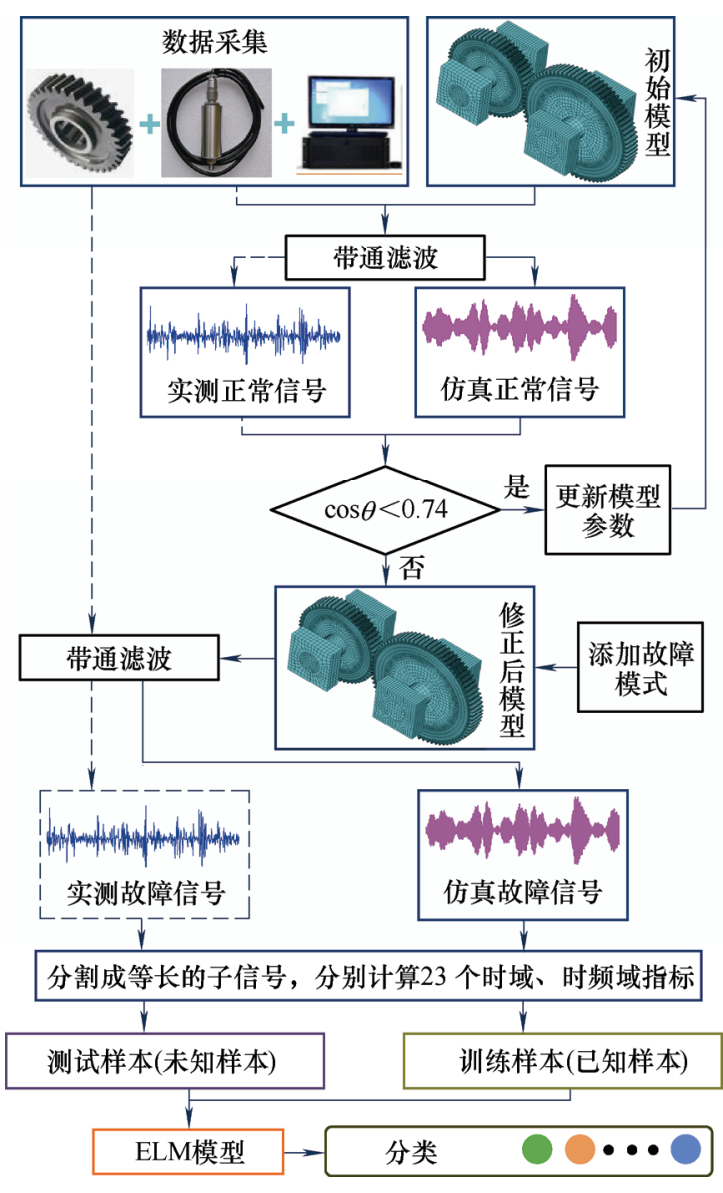

图 3 齿轮故障个性化诊断方法流程图

与第 2.1 节方法相比, 区别在于以下几点

(1) 实测与仿真故障信号均进行带通滤波处理。

（2）预处理利用时域统计量，通过计算原始信 号在时域与时频域上的 23 个指标, 构建训练样本和 测试样本, 分别为如表 1 所示的标准差 $x_{\text {std }}$ 、峭度 $x_{\text {kur }}$ 、峰值 $x_{\mathrm{p}}$ 、均方根幅值 $x_{\text {sra }}$ 、斜度 $x_{\text {ske }}$ 、波形指 数 $S F$ 、峰值因子 $P F$ 、脉冲因子 $I F$ 、均方根值 $x_{\mathrm{rms}}$ 等 9 个时域指标, 以及小波包分解分量的能量 $(8$ 个)、 集合经验模态分解分量的能量(6 个)两种时频指标:

第 $j$ 层中第 $i$ 个小波包分解分量的能量

$$
\mathrm{WPD}_{i}=\sum_{n=1}^{N}\left|x_{i}(n)\right|^{2} / \sum_{i=1}^{2^{j}} \sum_{n=1}^{N}\left|x_{i}(n)\right|^{2}
$$

式中, $x_{i}(n)$ 为小波包分解获得的第 $i\left(i=1,2, \cdots, 2^{j}\right)$ 个 
频带子信号, 令 $j=3$, 则小波包分解分量的能量 $\mathrm{WPD}_{i}(i=1,2, \cdots, 8)$ 。

第 $i$ 个集合经验模态分解分量的能量

$$
\operatorname{EEMD}_{i}=\sum_{n=1}^{N}\left|\operatorname{IMF}_{i}(n)\right|^{2} / \sum_{i=1}^{N I} \sum_{n=1}^{N}\left|\operatorname{IMF}_{i}(n)\right|^{2}
$$

式中, $\operatorname{IMF}_{i}(n)$ 为集合经验模态分解获得的第 $i$ 个 $(i=1,2, \cdots, N I)$ 本征模态分量, $N I$ 表示分解层数, 令 $N I=6$, 则集合经验模态分解分量的能量 $\operatorname{EEMD}_{i}(i=1$, $2, \cdots, 6)$ 。

(3) 分类器采用极限学习机。

(4) 由于齿轮传动系统信号相对复杂, 余弦相 似度阈值取 0.74 。

\section{3 有限元模拟驱动 $\mathrm{CNN}$ 的轴承一转子系统故障 个性化诊断方法}

与轴承、齿轮故障诊断方法类似, 针对轴承一 转子系统故障类型识别, 提出一种有限元模拟驱动 $\mathrm{CNN}$ 的轴承一转子系统故障个性化诊断方法, 方法 流程如图 4 所示。

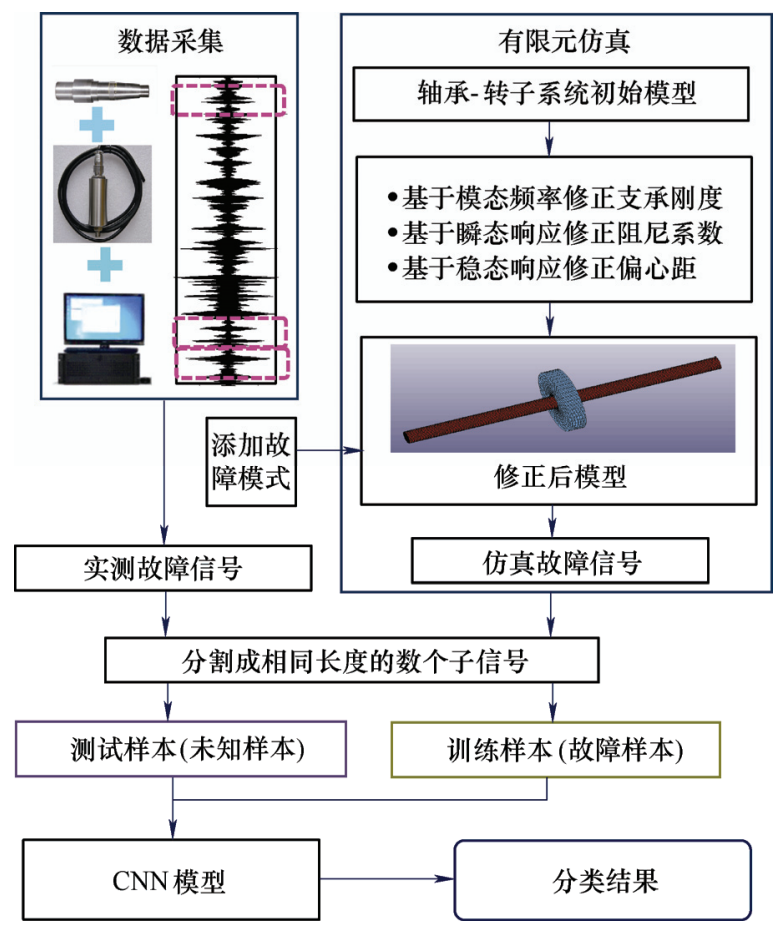

图 4 轴承一转子系统故障个性化诊断方法流程图

相对于轴承、齿轮而言, 轴承一转子系统待修 正的模型参数主要包括轴承支承刚度、阻尼系数、 加工误差引起的偏心距, 可分别进行修正: (1) 轴承转子系统一般为弱阻尼结构, 其中轴承支承刚度严 重影响结构的模态频率, 可以基于模态频率进行支 撑刚度的修正 ${ }^{[22]}$; (2) 阻尼对结构的模态频率影响 小，却与结构的瞬态响应在时域上的衰减程度成正 比, 通过比较仿真与实测的瞬态响应可修正阻尼系 数 ${ }^{[23]}$; (3) 偏心距是导致转子在正常运行状态下出
现振动响应的主要原因, 可计算仿真和实测的稳态 时域响的相似度修正转子的偏心距 ${ }^{[22]}$ 。此外, 由于 实验转子系统精度较高, 余弦相似度可取较大的值 (具体见第 3.3 节所述), 因此, $\mathrm{CNN}$ 直接处理原始 振动信号效果显著, 不需要对原始信号预处理。

\section{3 有限元仿真模型构建与修正}

\section{1 轴承}

\subsection{1 试验装置}

采用凯斯西储大学 (CWRU) 的滚动轴承数据 ${ }^{24]}$ 进行试验验证。试验对象为滚动轴承 SKF6 205, 几 何尺寸如图 5 所示, 轴承的转速为 $1797 \mathrm{r} / \mathrm{min}$, 采 样频率为 $6000 \mathrm{~Hz}$ 。
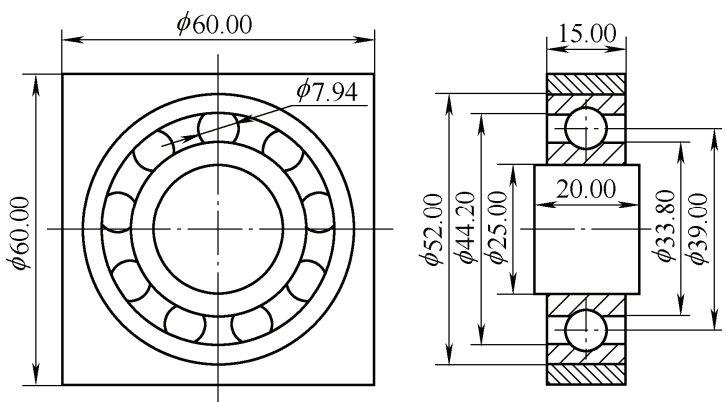

图 5 滚动轴承几何尺寸

\subsection{2 有限元仿真模型与修正}

利用有限元分析软件 ANSYS 建立轴承的有限 元模型, 如图 6a 所示, 采用 SOLID164 实体单元进 行网格剖分。为施加 $1797 \mathrm{r} / \mathrm{min}$ 的旋转载荷, 采用 SHELL163 壳体单元对内圈内表面进行网格剖分。 对于轴承的材料属性, 密度 $\rho=7860 \mathrm{~kg} / \mathrm{m}^{3}$, 弹性模 量 $E=2.06 \times 10^{11} \mathrm{~Pa}$, 泊松比 $\mu=0.3$ 。模型中包含两个 接触对, 分别为滚子与内、外圈之间的接触, 根据 文献[25-26], 设定摩擦因数分别为 0.016 和 0.02 。 为保证模型中的接触近似于刚性接触, 最大化用于 控制接触刚度的法向惩罚刚度因子 $\mathrm{FKN}^{[27]}$, 通过多 次仿真试验, 设定 FKN 的值为 0.12 , 同时计算出阻 尼比为 $0.0015^{[28]}$ 。对于轴承的约束条件, 内圈和转 轴同步旋转, 轴承座与轴承外圈固定连接, 并限制 轴承座表面节点的所有自由度。

然而作为影响轴承振动信号的敏感参数, 轴的 重力、偏心力与径向预紧应力均为需要识别的未知 量 ${ }^{[29]}$ 。因此, 在轴承有限元模型修正中, 重力、偏 心力及径向预紧应力为待修正参数。

按图 1 所示流程, 利用仿真与实测正常轴承振 动信号在时域上的余弦相似度来迭代求解, 需要确 定重力、偏心力及径向预紧应力的大致范围。由于 
轴结构尺寸未知, 可设一个较大修正范围 $[100 \mathrm{~N}$, $1000 \mathrm{~N}$ ], 均匀施加于轴线上的节点。根据文献[29] 给出的系列公式及轴承结构估算出一个大致值, 并 考虑轴承工作状态, 设定偏心载荷以应力的方式均 匀作用于内圈内表面的一半, 修正范围可取 $[0$, $0.2 \mathrm{MPa}$; 径向预紧力也以应力的方式均匀作用于 内圈内表面, 可大致设定修正范围为 $[0.5,2] \mathrm{MPa}$ 。

在重力、偏心力及径向预紧应力的大致范围内, 按一定步长迭代求解, 最终, 余弦相似度值为 $\cos (\theta)=0.605$ 时停止迭代, 得到对应的重力为 $500 \mathrm{~N}$ 、 偏心力 $0.12 \mathrm{MPa}$ 及径向预紧应力 $1 \mathrm{MPa}$, 对应的仿 真与实测信号的时域波形如图 7 所示。最终得到修 正后的轴承有限元模型参数, 如表 2 所示。

表 2 轴承有限元模型参数

\begin{tabular}{cc||cc}
\hline 接触参数 & 数值 & 载荷参数 & 数值 \\
\hline $\begin{array}{c}\text { 法向惩罚刚度因子 } \\
\text { FKN }\end{array}$ & 0.12 & 转轴重力载荷 $/ \mathrm{N}$ & 500 \\
$\begin{array}{c}\text { 滚子与外圈的摩擦 } \\
\text { 因数 }\end{array}$ & 0.016 & 偏心载荷 $/ \mathrm{MPa}$ & 0.12 \\
$\begin{array}{c}\text { 滚子内圈的摩擦 } \\
\text { 因数 }\end{array}$ & 0.02 & 旋转速度 $/(\mathrm{r} / \mathrm{min})$ & 1797 \\
黏滞阻尼比 & 0.0015 & $\begin{array}{c}\text { 径向预紧载荷 } \\
/ \mathrm{MPa}\end{array}$ & 1 \\
\hline
\end{tabular}

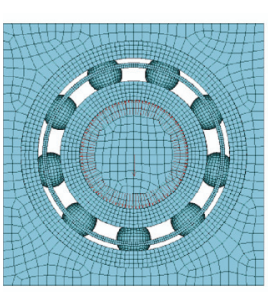

(a) 有限元模型

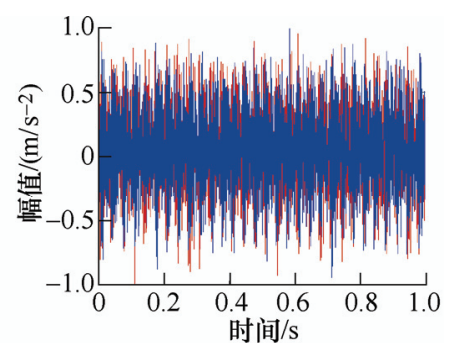

(b) 仿真与实测信号的时域响应
图 6 滚动轴承模型及仿真与实测信号

\section{2 齿轮}

\subsection{1 试验装置}

图 7 所示为一单级齿轮传动系统, 其几何尺寸 如图 8 所示。表 3 与表 4 分别给出了齿轮与圆柱滚 子轴承的几何与材料参数。

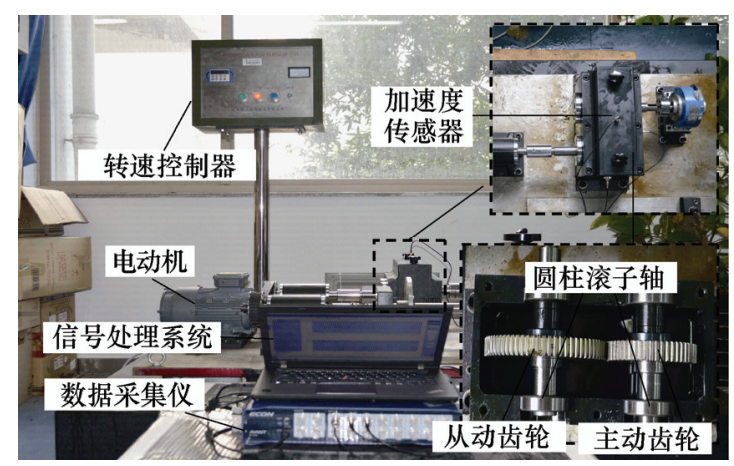

图 7 齿轮传动系统试验装置
输入转速为 $1475 \mathrm{r} / \mathrm{min}, 5 \mathrm{~N} / \mathrm{m}$ 的负载扭矩作 用在从动齿轮轴上, 从齿轮箱体上采集加速度信号, 采样频率为 $5120 \mathrm{~Hz}$ 。

表 3 齿轮几何、材料参数

\begin{tabular}{cc||cc}
\hline 材料参数 & 数值 & 几何参数 & 数值 \\
\hline 材料密度 $/\left(\mathrm{kg} / \mathrm{m}^{3}\right)$ & 7850 & 主动齿轮齿数 & 55 \\
弹性模量 $/ \mathrm{Pa}$ & $2.06 \times 10^{11}$ & 从动齿轮齿数 & 75 \\
泊松比 & 0.3 & 模数 $/ \mathrm{mm}$ & 2 \\
\hline
\end{tabular}

表 4 轴承几何、材料参数

\begin{tabular}{cc||cc}
\hline 材料参数 & 数值 & 几何参数 & 数值 \\
\hline 弹性模量 $/ \mathrm{Pa}$ & $2.06 \times 10^{11}$ & 内滚道直径 $/ \mathrm{m}$ & 0.03 \\
$\begin{array}{c}\text { 泊松比 } \\
\begin{array}{c}\text { 滚子有效接触 } \\
\text { 长度 } / \mathrm{m}\end{array}\end{array}$ & 0.3 & 滚子直径 $/ \mathrm{mm}$ & 7.5 \\
\hline
\end{tabular}

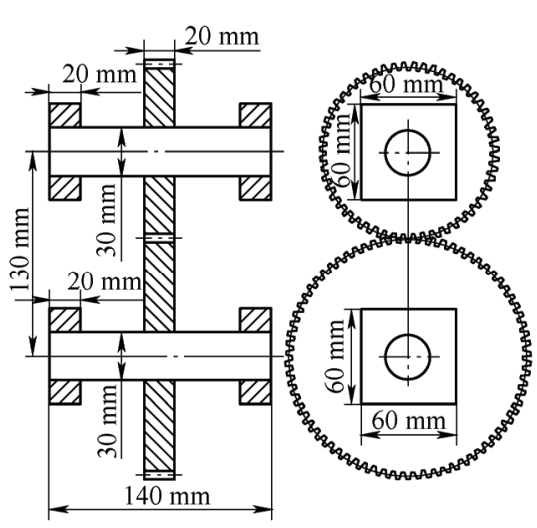

(a) 装配图

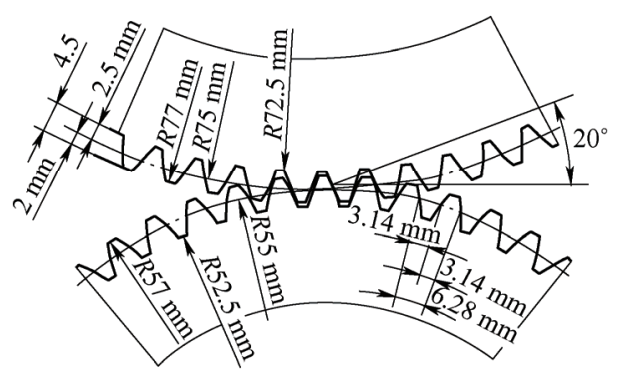

(b) 啮合图

图 8 齿轮传动系统几何参数

\subsection{2 有限元仿真模型与修正}

图 9 所示为一单级齿轮传动系统的有限元模 型。模型中通过设定 4 个特性相同的接触对 $C_{1} 、 C_{2}$ 、 $C_{3} 、 C_{4}$ 模拟 4 个轴承支撑刚度 $\mathrm{FKN}_{1}$ 与支撑阻尼比 $\xi_{1} ; 1$ 个接触对 $C_{5}$ 模拟齿轮接触摩擦系数 $\mu$ 、啮合 刚度 $\mathrm{FKN}_{2}$ 与啮合阻尼比 $\xi_{2}$ 。采用 SOLID164 实体 单元进行网格剖分, 同时采用 SHELL163 壳体元件 剖分输入轴(主动齿轮轴)的表面以施加 $1475 \mathrm{r} / \mathrm{min}$ 的旋转载荷, 并对输出轴(从动齿轮轴)施加 $5 \mathrm{~N} / \mathrm{m}$ 的负载扭矩。 
根据文献[30-32], 可设定系统中圆柱滚子轴承 $\mathrm{FKN}_{1}=0.12 、 \xi_{1}=0.09$, 齿轮啮合的摩擦因数 $\mu=0.08$ 。

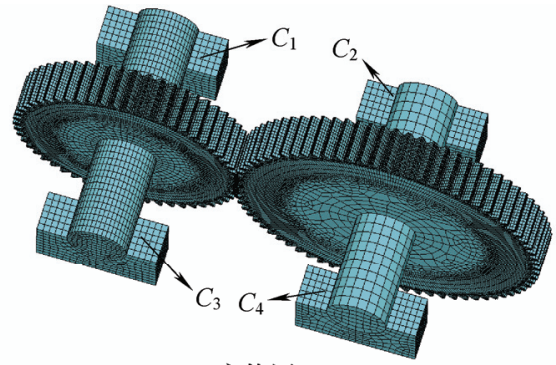

(a) 立体图

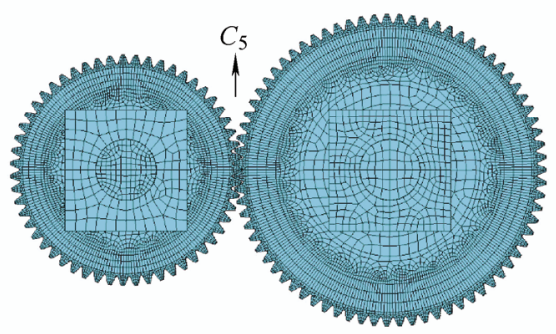

(b) 主视图

图 9 齿轮传动系统有限元模型

齿轮的啮合刚度 $\mathrm{FKN}_{2}$ 和啮合阻尼 $\xi_{2}$ 为影响齿 轮啮合时振动响应的未知敏感参数, 其变化导致齿 轮振动以其啮合频率为中心的边频带。因此, 为减 小强烈的噪声, 采用带通滤波进行信号预处理, 考 虑到齿轮啮合频率为 $1352 \mathrm{~Hz}$, 主动轮和从动轮的 旋转频率分别为 $24.5 \mathrm{~Hz}$ 和 $18 \mathrm{~Hz}$, 可设定带通滤波 器的中心频率 $f_{c}=1352 \mathrm{~Hz}$, 带宽为 $200 \mathrm{~Hz}$, 对仿真 与实测信号进行带通滤波消噪, 按图 3 所示模型修 正步骤进行迭代, 得到余弦相似度 $\cos (\theta)=0.742$, 对 应的 $\mathrm{FKN}_{2}=0.15 、 \xi_{2}=0.03$ 。因此, 确定修正后齿轮 有限元模型参数如表 5 所示。

表 5 齿轮有限元模型参数

\begin{tabular}{cl}
\hline 模型参数 & 数值 \\
\hline 轮齿接触摩擦因数 $\mu$ & 0.08 \\
轴承支撑刚度 $\mathrm{FKN}_{1}$ & 0.12 \\
轴承支撑阻尼比 $\xi_{1}$ & 0.09 \\
齿轮啮合刚度 $\mathrm{FKN}_{2}$ & 0.15 \\
齿轮啮合阻尼比 $\xi_{2}$ & 0.03 \\
\hline
\end{tabular}

\section{3 轴承一转子系统}

\subsection{1 试验装置}

图 10 所示为轴承-转子系统故障模拟系统, 其 材料参数如表 6 所示, 几何模型如图 11 所示。为减 少计算时间, 利用有限元分析软件 ANSYS 建立一 维有限元模型, 轴承刚度系数 $K_{x} 、 K_{y}$, 阻尼系数 $C_{x} 、 C_{y}$, 转子偏心距 $e_{\text {。 }}$
表 6 转子材料参数

\begin{tabular}{ccc}
\hline 材料参数 & 转轴 & 质量圆盘 \\
\hline 材料密度 $/\left(\mathrm{kg} / \mathrm{m}^{3}\right)$ & 7850 & 2700 \\
弹性模量 $/ \mathrm{Pa}$ & $2.06 \times 10^{11}$ & $7 \times 10^{10}$ \\
泊松比 & 0.3 & 0.34 \\
质量 $/ \mathrm{kg}$ & 2.219 & 0.627 \\
\hline
\end{tabular}

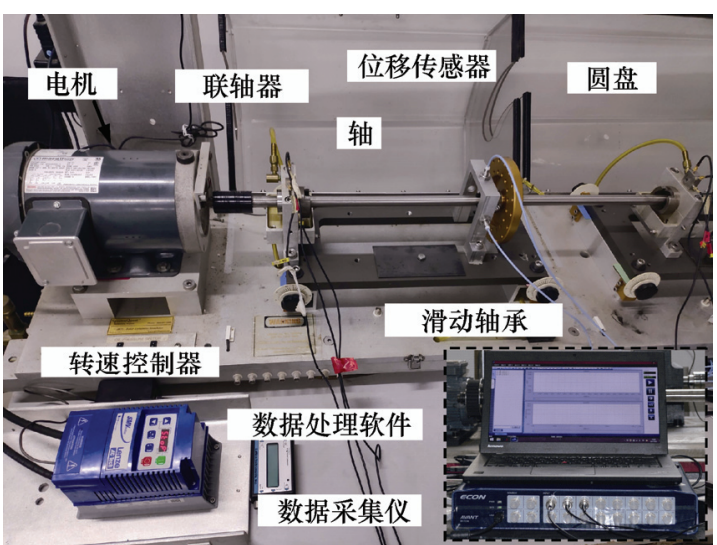

图 10 轴承-转子系统试验装置

利用 BEAM188 单元对转子进行网格剖分, 分 别采用 MASS2 与 COMBINE14 模拟圆盘与两端的 滑动轴承。根据轴承一转子的实际工作情况, 对 COMBINE14 单元端点(轴承外圈)设置全约束。

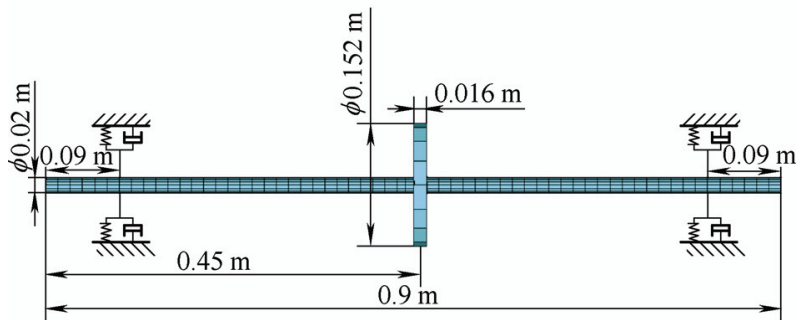

(a) 转子

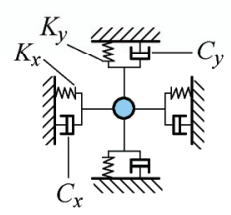

(b) 轴承

图 11 轴承-转子系统模型

为保证仿真信号能有效代替实测信号, 将用于 模拟滑动轴承的刚度、阻尼系数以及偏心距作为敏 感参数, 分别基于模态频率 ${ }^{[22]} 、$ 瞬态响应 ${ }^{[23]}$ 和稳态 时域响应 ${ }^{[23]}$ 进行修正。

模态频率与瞬态响应分析采用力锤法进行实验 模态分析。如图 12 所示, 分别在转子的 $X$ 和 $Y$ 方 向进行实验模态测试, 设定信号的采样频率为 $24 \mathrm{kHz}$, 在 $X$ 方向, 敲击 $A_{1}$ 点, 从 $A_{2} 、 A_{3}$ 和 $A_{4}$ 点 
拾取加速度瞬态响应信号; 在 $Y$ 方向; 在 $B_{1}$ 点敲 击, 在 $B_{2} 、 B_{3} 、 B_{4}$ 点拾取。

(1) 刚度系数

通过 $A_{2} 、 A_{3}$ 和 $A_{4}$, 以及 $B_{2} 、 B_{3} 、 B_{4}$ 点拾取的 瞬态响应频谱分析及相互比对, 可获得转子沿 $X$ 方 向的前三阶弯曲模态频率: $174 \mathrm{~Hz} 、 261 \mathrm{~Hz}$ 与 $2160 \mathrm{~Hz}$, 沿 $Y$ 方向的前三阶弯曲模态频率:

$170 \mathrm{~Hz} 、 252 \mathrm{~Hz}$ 与 $2096 \mathrm{~Hz}$ 。设定 $K_{x}$ 与 $K_{y}$ 的取值 范围 $\left[15 \times 10^{7} \mathrm{~N} / \mathrm{m}, 30 \times 10^{7} \mathrm{~N} / \mathrm{m}\right]^{[22]}$, 由于修正量仅 为沿 $X$ 方向或 $Y$ 方向的三阶频率，故可设 $\cos (\theta)=1$, 迭代求解, 可得 $K_{x}=23.5 \times 10^{7} \mathrm{~N} / \mathrm{m} 、 K_{y}=20.5 \times 10^{7}$ $\mathrm{N} / \mathrm{m}$, 对应的 6 个模态频率如表 7 所示。

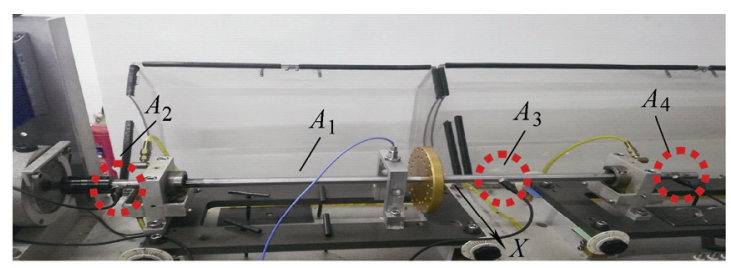

(a) $X$ 方向

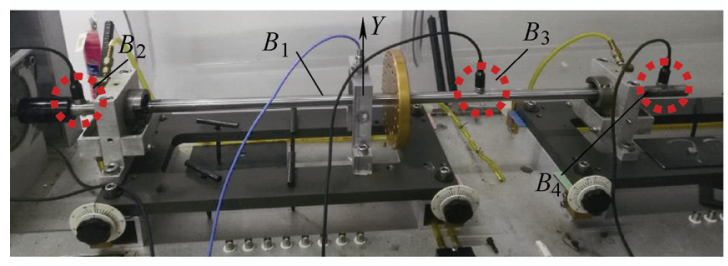

(b) $Y$ 方向

图 12 转子试验模态分析

表 7 转子支承刚度

\begin{tabular}{|c|c|c|c|c|c|c|c|}
\hline \multirow{2}{*}{\multicolumn{2}{|c|}{ 支承刚度 }} & \multicolumn{6}{|c|}{ 弯曲模态频率 } \\
\hline & & \multicolumn{2}{|c|}{ 一阶/Hz } & \multicolumn{2}{|c|}{ 二阶 $/ \mathrm{Hz}$} & \multicolumn{2}{|c|}{ 三阶/Hz } \\
\hline$K_{x} /(\mathrm{N} / \mathrm{m})$ & $K_{y} /(\mathrm{N} / \mathrm{m})$ & $X$ & $Y$ & $X$ & $Y$ & $X$ & $Y$ \\
\hline $23.5 \times 10^{7}$ & $20.5 \times 10^{7}$ & 174.5 & 169.9 & 264.2 & 248.9 & 2152.9 & 2099.5 \\
\hline
\end{tabular}

(2) 阻尼系数

设定 $C_{x}$ 与 $C_{y}$ 的取值范围 $[1000 \mathrm{~N} \cdot \mathrm{s} / \mathrm{m}, 2000$ $\mathrm{N} \cdot \mathrm{s} / \mathrm{m}]^{[23]}, \cos (\theta)$ 阈值为 0.83 , 固定 $K_{x}=23.5 \times 10^{7}$ $\mathrm{N} / \mathrm{m} 、 K_{y}=20.5 \times 10^{7} \mathrm{~N} / \mathrm{m}$, 迭代求解 $C_{x}$ 与 $C_{y}$, 最终 确定 $C_{x}=1500 \mathrm{~N} \cdot \mathrm{s} / \mathrm{m}$ 和 $C_{y}=1700 \mathrm{~N} \cdot \mathrm{s} / \mathrm{m}$ 。图 13 所示 为修正后 $A_{2} 、 B_{2}$ 点上仿真与实测瞬态响应波形比 较结果。

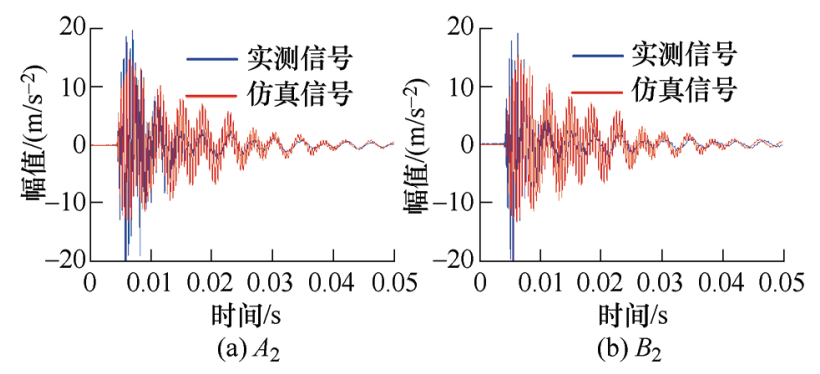

图 13 瞬态响应时域波形
(3) 偏心距

利用稳态时域响应确定偏心距 $e$, 设定转速为 $600 \mathrm{r} / \mathrm{min}$, 采样频率 $f_{s}=3000 \mathrm{~Hz}$, 通过图 10 所示的 位移传感器获取转子振动信号。转子离心力

$$
\begin{aligned}
& F_{\mathrm{e}}(x)=m \cdot e \cdot \omega^{2} \cos (\omega \cdot t) \\
& F_{\mathrm{e}}(y)=m \cdot e \cdot \omega^{2} \sin (\omega \cdot t)
\end{aligned}
$$

式中, $m=2.846 \mathrm{~kg}$ 为转子的质量; $\omega$ 为角速度 $(\mathrm{rad} / \mathrm{s})$ 。 $e$ 可选一较大范围 $\left[0.001 \times 10^{-3} \mathrm{~m}, 0.3 \times 10^{-3} \mathrm{~m}\right]$ 。固定 $K_{x}=23.5 \times 10^{7} \mathrm{~N} / \mathrm{m} 、 K_{y}=20.5 \times 10^{7} \mathrm{~N} / \mathrm{m}, C_{x}=1500$ $\mathrm{N} \cdot \mathrm{s} / \mathrm{m} 、 C_{y}=1700 \mathrm{~N} \cdot \mathrm{s} / \mathrm{m}$, 迭代调整 $e$, 仿真获得缓变 的仿真位移信号, 设置阈值 $\cos (\theta)=1$, 确定 $e=0.164 \times 10^{-3} \mathrm{~m}$ 。

\section{4 诊断效果分析}

\section{1 轴承}

\subsection{1 仿真故障样本生成}

根据 CWRU 提供的轴承信号 ${ }^{[24]}$, 取 6 种故障 模式, 用孔径为 $d$ 、深度统一为 $h=0.2794 \mathrm{~mm}$ 的盲 孔模拟: 外圈 $(d=0.1778 \mathrm{~mm})$ 、外圈 $(d=0.5334 \mathrm{~mm})$ 、 内圈 $(d=0.1778 \mathrm{~mm})$ 、内圈 $(d=0.5334 \mathrm{~mm})$ 、滚动体 $(d=0.1778 \mathrm{~mm})$ 、滚动体 $(d=0.5334 \mathrm{~mm})$, 分别简记 为 $T_{1} 、 T_{2} 、 T_{3} 、 T_{4} 、 T_{5} 、 T_{6}$, 如图 14 所示。利用修 正后的轴承有限元模型, 通过计算生成 6 种故障模 式的仿真故障信号, 信号长度取 12000 个数据点 $(2 \mathrm{~s})$, 如图 15 所示。

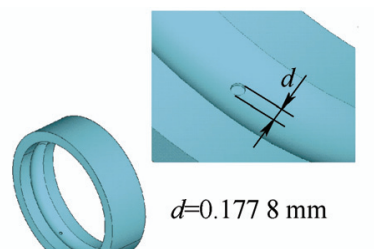

(a) $T_{1}$

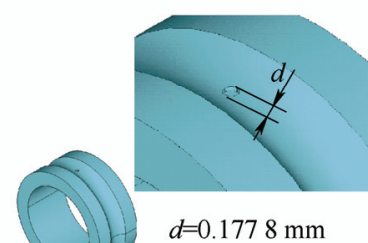

(c) $T_{3}$

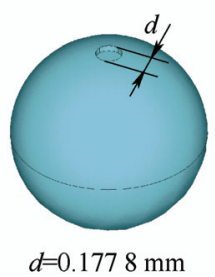

(e) $T_{5}$

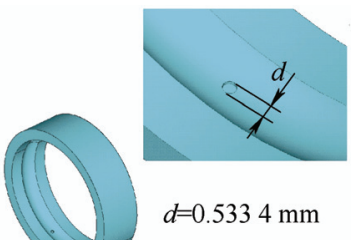

(b) $T_{2}$

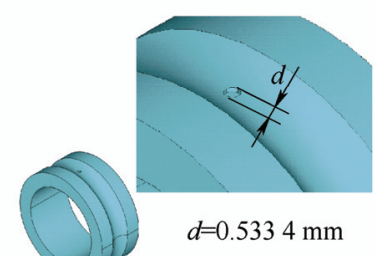

(d) $T_{4}$

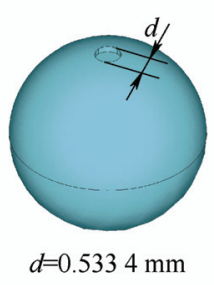

(f) $T_{6}$

图 14 故障轴承几何模型 


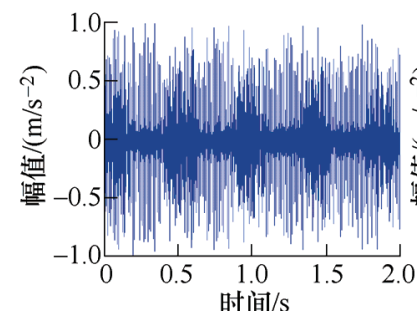

(a) 故障模式 $T_{1}$

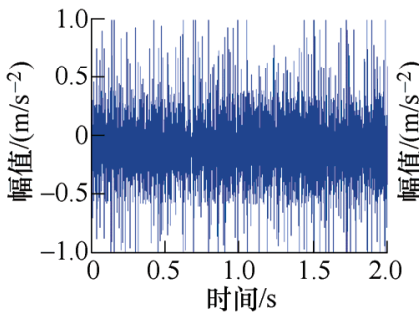

(c) 故障模式 $T_{3}$

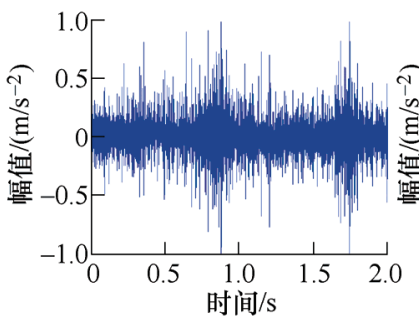

(e) 故障模式 $T_{5}$

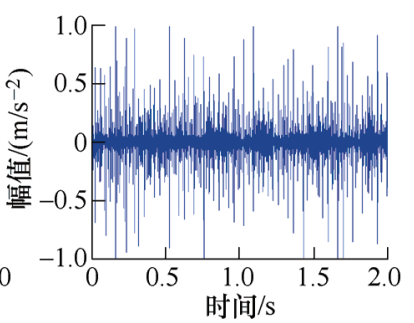

(b) 故障模式 $T_{2}$

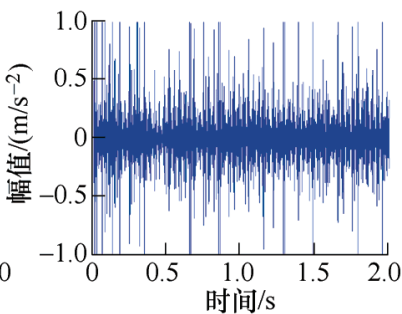

(d) 故障模式 $T_{4}$

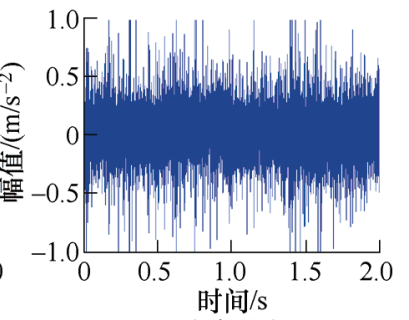

(f) 故障模式 $T_{6}$
图 15 仿真信号时域响应

为生成故障训练样本, 将每个仿真故障信号在 时域上分成 40 个等长度的子信号, 计算每个子信号 如表 1 所示的 16 个时域指标。因此, 对于每种故障 模式 $T_{i}(i=1,2, \cdots, 6)$ 共有 40 个训练样本, 即: $16 \times 40$ 个时域特征指标集。

\subsection{2 基于支持向量机识别故障}

以 CWRU 提供的 $T_{1} 、 T_{2} 、 T_{3} 、 T_{4} 、 T_{5} 、 T_{6}$ 这 6 种故障信号作为待诊断的测试样本, 与仿真生成的 训练样本一起, SVM 输入径向基函数容错率 $C=10$ 、 基函数半径 $g=0.807$ 的 SVM 模型, 故障分类结果如 表 8 所示。为评估个性化诊断效果, 表 8 还给出了 以 $T_{1} 、 T_{2} 、 T_{3} 、 T_{4} 、 T_{5} 、 T_{6}$ 这 6 种测试故障信号同 时作为训练与测试样本的分类结果 (一般用于验证 人工智能模型的可用性), 由于训练样本与测试样本 完全一样, 因此作为分类准确度的 SVM 基准(在表 8 中简称为基准)评估本文方法有效性。值得指出的 是: 由于缺乏故障样本, 大量已发表的人工智能模 型诊断论文均停留在利用已有（有限且相同）的训 练样本与测试样本, 优化人工智能模型, 获得仅适 用于已有故障样本的 “最佳” 模型, 而实际使用中 效果较差。

从表 8 可知, 在个性化诊断中, 外圈故障的分 类准确率分别为 $90 \% 、 92.5 \%$, 内圈故障的分类准 确率分别为 $87.5 \% 、 87.5 \%$, 滚动体故障的分类准确
率分别为 $85 \% 、 82.5 \%$, 略低于 SVM 基准分类准确 率。与 SVM 基准相比, 个性化诊断准确率相对误 差为 $2.2 \% \sim 12.8 \%$, 这足以验证基于有限元模拟驱 动 SVM 的轴承故障个性化诊断方法具有可行性。

表 8 SVM 故障分类结果

\begin{tabular}{ccccccc}
\hline 故障模式 & 训练样本 & 测试样本 & 标签 & 个性化(\%) & 基准(\%) & 误差(\%) \\
\hline$T_{1}$ & 40 & 40 & 1 & 90 & 92 & 2.2 \\
$T_{2}$ & 40 & 40 & 2 & 92.5 & 95 & 2.6 \\
$T_{3}$ & 40 & 40 & 3 & 87.5 & 95 & 7.9 \\
$T_{4}$ & 40 & 40 & 4 & 87.5 & 90 & 7.9 \\
$T_{5}$ & 40 & 40 & 5 & 85 & 97.5 & 12.8 \\
$T_{6}$ & 40 & 40 & 6 & 82.5 & 87.5 & 5.7 \\
\hline
\end{tabular}

\section{2 齿轮}

4.2.1 仿真故障样本生成

根据齿轮的常见故障, 设计仿真如图 16 所示的 五种故障模式: 主动齿轮轮齿裂纹 $G_{1}$ 、主动齿轮齿 面剥落 $G_{2}$ 、主动齿轮断齿 $G_{3}$ 、主动齿轮齿面剥落 和从动齿轮断齿 $G_{4}$ 、主动齿轮和从动齿轮同时断齿 $G_{5}$ 。对应的实验故障齿轮如图 17 所示。

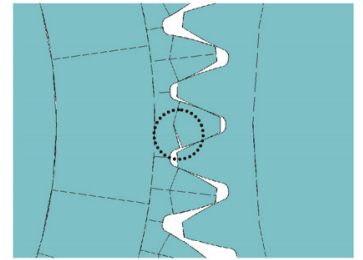

(a) 故障模式 $G_{1}$

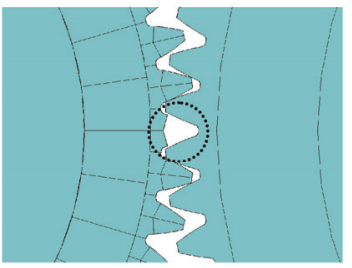

(c) 故障模式 $G_{3}$

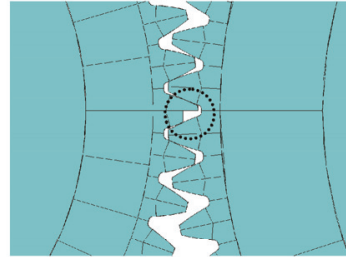

(b) 故障模式 $G_{2}$

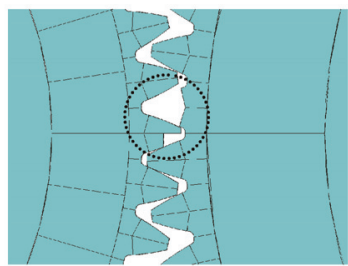

(d) 故障模式 $G_{4}$

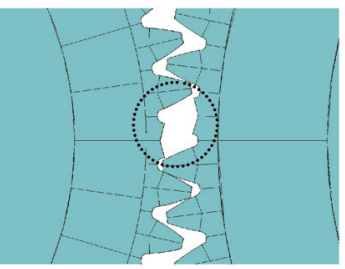

(e) 故障模式 $G_{5}$

图 16 齿轮故障模型

利用修正后的正常齿轮有限元模型, 考虑 $G_{1}$, $G_{2}, G_{3}, G_{4}, G_{5}$ 五种故障, 模拟获得仿真故障信号 并进行与 3.2.2 节一样的滤波处理, 时域波形如 图 18 所示, 信号长度为 $12000(2.34 \mathrm{~s})$ 。

为生成故障训练样本, 将每个滤波后的仿真信 号在时域上等长度地分成 40 个子信号, 计算每个子 信号如表 2 所示的 23 个时域、时频域指标。因此, 
对于齿轮中的每种故障模式 $G_{i}(i=1,2, \cdots, 5)$, 共有 40 个训练样本, 即: $23 \times 40$ 个时域特征指标集。

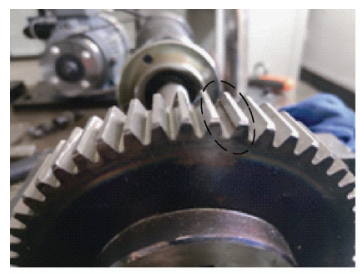

(a) 主动轮齿裂纹

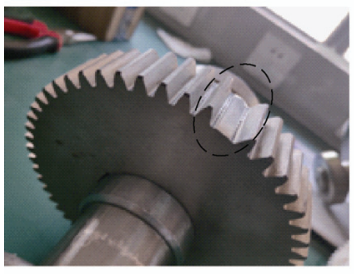

(c) 主动齿轮断齿

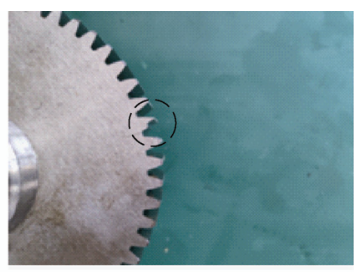

(b) 主动轮齿剥落

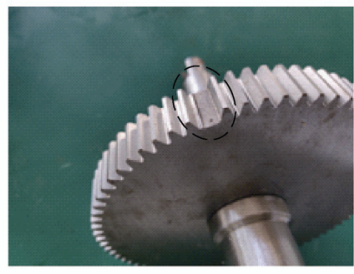

(d) 从动齿轮断齿
图 17 故障齿轮

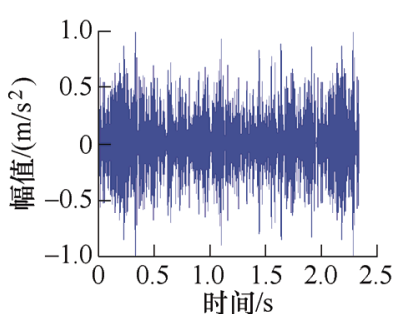

(a) 故障模式 $G_{1}$

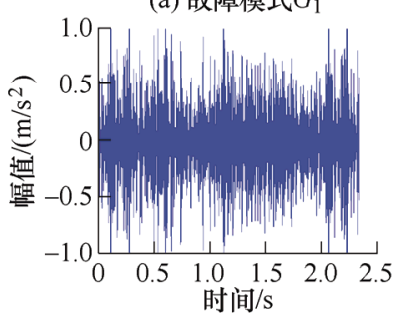

(c) 故障模式 $G_{3}$

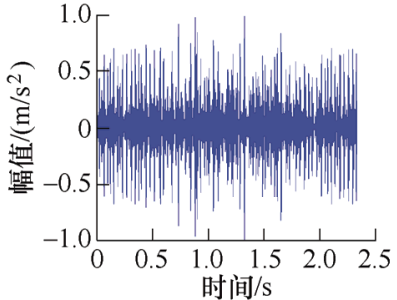

(b) 故障模式 $G_{2}$

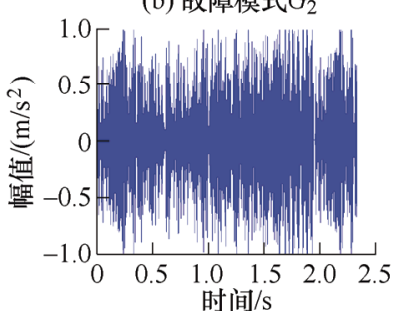

(d) 故障模式 $G_{4}$

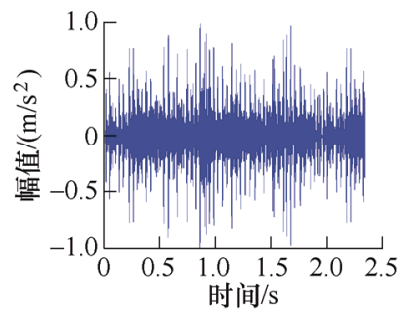

(e) 故障模式 $G_{5}$

图 18 滤波后的仿真信号

\subsection{2 基于极限学习机识别故障}

将故障训练样本输入 ELM 模型中进行智能诊 断模型的训练, 训练过程中, ELM 的隐含层节点个 数为 25 , 训练次数为 5000 。而 ELM 模型的 5 种测 试样本均来为实测信号, 测试样本的生成方式与训 练样本相同, 故障分类结果如表 9 所示。为评估个 性化诊断效果, 表 9 还给出了 ELM 模型分类中以 $G_{1} 、 G_{2} 、 G_{3} 、 G_{4} 、 G_{5}$ 这 5 种测试故障信号同时作为 训练与测试样本的分类结果(简称 ELM 基准)。从
表 9 可知, 在个性化诊断中, $G_{1} 、 G_{2} 、 G_{3} 、 G_{4} 、 G_{5}$ 这 5 种齿轮故障分类准确率分别为 $80 \%$ 、 $87.5 \%$ 、 $80 \% 、 90 \% 、 85 \%$, 略低于 ELM 基准分类准确率。 与 ELM 基准相比, 个性化诊断准确率相对误差为 $5.2 \% \sim 11.1 \%$ 。由于仿真信号是通过计算简化的有 限元模型所获得的, 难以完全匹配试验信号, 但是 这足以验证基于有限元模拟驱动 ELM 的齿轮故障 个性化诊断方法具有可行性, 并能有效地克服了工 程应用中缺乏故障样本的根本性难题。

表 9 ELM 故障分类结果

\begin{tabular}{ccccccc}
\hline 故障模式 & 训练样本 & 测试样本 & 标签 & 个性化 $(\%)$ & 基准 $(\%)$ & 误差 $(\%)$ \\
\hline$G_{1}$ & 40 & 40 & 1 & 80 & 85 & 5.8 \\
$G_{2}$ & 40 & 40 & 2 & 87.5 & 92.5 & 5.4 \\
$G_{3}$ & 40 & 40 & 3 & 80 & 90 & 11.1 \\
$G_{4}$ & 40 & 40 & 4 & 90 & 95 & 5.2 \\
$G_{5}$ & 40 & 40 & 6 & 85 & 95 & 10.5 \\
\hline
\end{tabular}

\section{3 轴承一转子系统}

\subsection{1 仿真故障样本生成}

如图 19 所示, 设计故障: 不平衡、不对中及转 子碰摩三种状态下的 9 种故障类型, 在仿真与实验 转子设定转子转速 $600 \mathrm{r} / \mathrm{min}$, 采样频率 $f_{\mathrm{s}}=3000 \mathrm{~Hz}$ 。

(1) 不平衡故障

将转子不平衡产生的离心力 $F_{\delta}(x)$ 和 $F_{\delta}(y)$ 施加 于圆盘的 $X$ 方向和 $Y$ 方向，可计算 ${ }^{[33]}$

$$
\begin{aligned}
& F_{\delta}(x)=m_{\delta} \cdot \delta \cdot \omega^{2} \cos (\omega t) \\
& F_{\delta}(y)=m_{\delta} \cdot \delta \cdot \omega^{2} \sin (\omega t)
\end{aligned}
$$

式中, $m_{\delta}$ 为的偏心质量, $\delta$ 为偏心距, $\omega$ 为转子角 速度。实验中考虑三种严重程度不同的不平衡故障 $R_{1} 、 R_{2}$ 和 $R_{3}$, 对应的 $m_{\delta}$ 分别为 $0.007 \mathrm{~kg} 、 0.015 \mathrm{~kg}$ 和 $0.023 \mathrm{~kg}$, 其中 $\delta$ 为常数, 为 $0.052 \mathrm{~m}$ 。

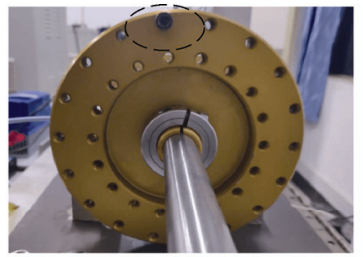

(a) 不平衡

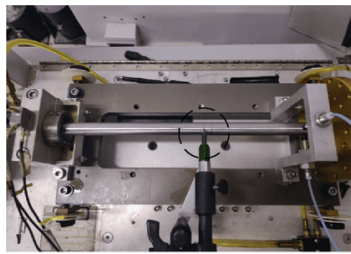

(c) 转子碰摩

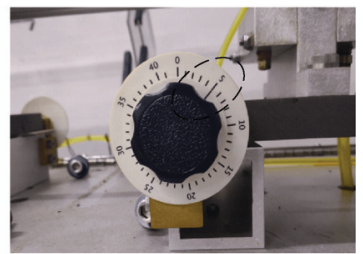

(b) 不对中

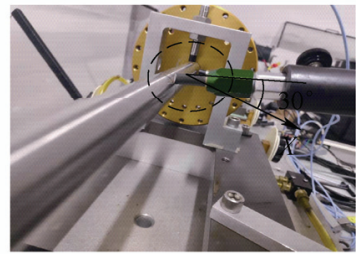

(d) 转子碰摩
图 19 试验中转子的三种故障状态

(2) 不对中故障

对于不对中故障, 只考虑联轴器与转子之间的 
平行不对中, 将不对中的激振力 $F_{\mathrm{m}}(x)$ 与 $F_{\mathrm{m}}(y)$ 施加 于转子最左端的节点上, 可由下式计算 ${ }^{[33]}$

$$
\begin{aligned}
& F_{\mathrm{m}}(x)=m_{c} \cdot c_{e} \cdot \omega^{2} \cos (2 \cdot \omega \cdot t) \\
& F_{\mathrm{m}}(y)=m_{c} \cdot c_{e} \cdot \omega^{2} \sin (2 \cdot \omega \cdot t)
\end{aligned}
$$

式中, $m_{c}=0.382 \mathrm{~kg}$ 为联轴器的质量, $c_{e}$ 为转子的平 行不对中距离。考虑三种严重程度不同的不对中故 障 $R_{4} 、 R_{5}$ 和 $R_{6}$, 对应的 $c_{e}$ 分别为 $0.127 \times 10^{-3} \mathrm{~m}$ 、 $0.254 \times 10^{-3} \mathrm{~m}$ 和 $0.508 \times 10^{-3} \mathrm{~m}$ 。

\section{(3) 转子碰摩故障}

根据文献[33], 转子碰摩故障产生的冲击力可 表示为与转速和碰撞角度相关的三角波形, 如图 20 所示, 碰撞位置与 $X$ 方向夹角为 $30^{\circ}$ 时, 如图 $19 \mathrm{~d}$ 所示, 故障在 $X$ 方向产生 $f_{x}=3 \mathrm{~N}$ 的力、 $Y$ 方向产生 $f_{y}=1.5 \mathrm{~N}$ 的力。考虑碰撞角度 $30^{\circ}$ 时的三种严重程 度不同的碰摩故障 $R_{7}: f_{x}=3 \mathrm{~N} 、 f_{y}=1.5 \mathrm{~N}, R_{8}: f_{x}=8$ $\mathrm{N} 、 f_{y}=4 \mathrm{~N}, R_{9}: f_{x}=12 \mathrm{~N} 、 f_{y}=6 \mathrm{~N}$ 。

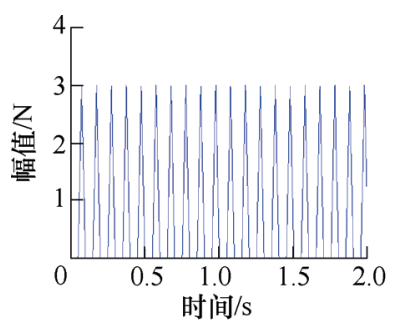

(a) $X$ 方向

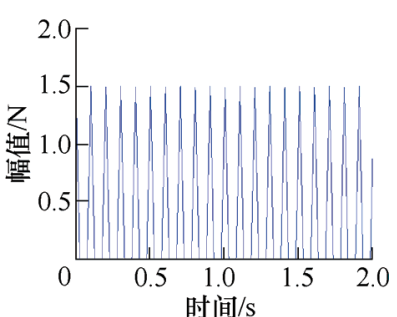

(b) $Y$ 方向
图 20 转子碰摩故障激振力

基于修正的正常转子有限元模型, 分别施加 9 种故障引起的激振力进行仿真分析, 激励的位置如 图 19c 所示, 距离圆盘左端 $0.15 \mathrm{~m}$ 处。在图 10 所 示位移传感器位置, 提取轴承一转子系统有限元分 析模型中 $X$ 方向的位移仿真信号。图 21 给出了 $R_{6}$ 、 $R_{7} 、 R_{8} 、 R_{9}$ 这四种转子故障仿真信号前 $2 \mathrm{~s}$ 数据。

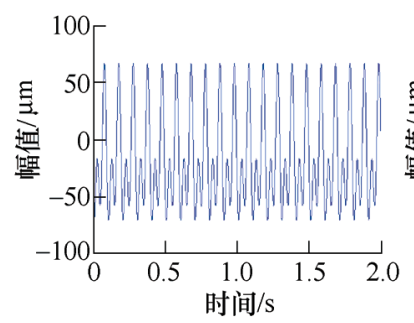

(a) $R_{6}$

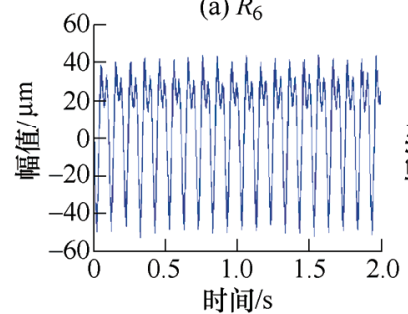

(c) $R_{8}$

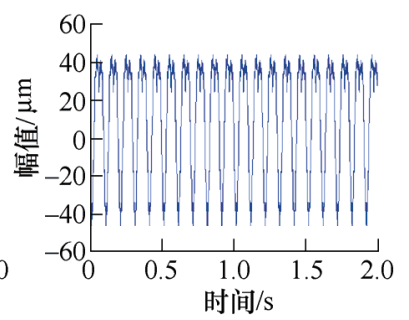

(b) $R_{7}$

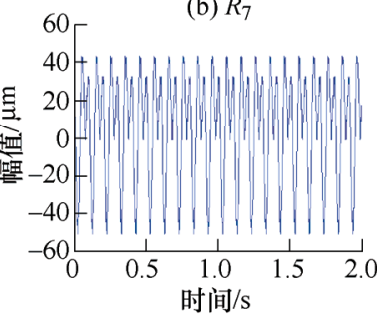

(d) $R_{9}$
图 21 转子 4 种故障位移仿真信号
作为故障训练样本, 每个仿真信号的长度为 72000 个数据点 $(20 \mathrm{~s})$, 按 CNN 模型输入形式 $20 \times 20$ 的矩阵, 直接分割原始信号, 得到 9 种故障训练样 本输入矩阵个数为: $180 \times 9$ 。

\subsection{2 基于卷积神经网络识别故障}

将故障训练样本输入 CNN 模型中进行智能诊 断模型的训练, 训练过程中, $\mathrm{CNN}$ 模型的主要参数 如表 10 所示。CNN 模型的 9 种测试样本均来为实 测信号, 测试样本的生成方式与训练样本相同。 表 11 给出了 9 类故障分类结果 $R_{1} \sim R_{9}$ 分类准确率分 别为 $88 \% 、 92 \% 、 90 \% 、 100 \% 、 96 \% 、 95 \% 、 91 \%$ 、 97\%、92\%, 与验证 CNN 模型的故障分类准确率的 $\mathrm{CNN}$ 基准相比，相对误差 $0 \% \sim 6.3 \%$ 。这验证了基 于有限元模拟驱动 $\mathrm{CNN}$ 的轴承-转子系统故障个性 化诊断方法有效性。

表 $10 \mathrm{CNN}$ 模型的主要参数

\begin{tabular}{cc}
\hline $\mathrm{CNN}$ 各层参数 & 数值 \\
\hline 输入层 & 输入图片大小: $20 \times 20$ \\
卷积层 $C_{1}$ & 卷积核大小: 5 , 特征图片张数: 6 \\
下采样层 $S_{1}$ & 尺度: 2 , 特征图片张数: 6 \\
卷积层 $C_{2}$ & 卷积核大小: 5 , 特征图片张数: 12 \\
下采样层 $S_{2}$ & 尺度: 2, 特征图片张数: 12 \\
其他参数 & 最大迭代次数: 500 , 学习速率: 1 , 批大小: 30 \\
\hline
\end{tabular}

表 $11 \mathrm{CNN}$ 故障分类结果

\begin{tabular}{ccccccc}
\hline 故障模式 & 训练样本 & 测试样本 & 标签 & 个性化(\%) & 基准(\%) & 误差(\%) \\
\hline$R_{1}$ & 180 & 180 & 1 & 88 & 94 & 6.3 \\
$R_{2}$ & 180 & 180 & 2 & 92 & 89 & 3.4 \\
$R_{3}$ & 180 & 180 & 3 & 90 & 93 & 3.2 \\
$R_{4}$ & 180 & 180 & 4 & 100 & 100 & 0 \\
$R_{5}$ & 180 & 180 & 5 & 96 & 100 & 4.0 \\
$R_{6}$ & 180 & 180 & 6 & 95 & 97 & 2.1 \\
$R_{7}$ & 180 & 180 & 7 & 91 & 94 & 3.2 \\
$R_{8}$ & 180 & 180 & 8 & 97 & 100 & 3.0 \\
$R_{9}$ & 180 & 180 & 9 & 92 & 97 & 5.1 \\
\hline
\end{tabular}

本节中给出的轴承、齿轮、轴承一转子系统三 个具体诊断实例, 基于机械传动系统故障诊断的个 性化诊断原理, 随机地采用 SVM、ELM、CNN 为 分类器, 可成功诊断出多类故障。与分类准确度最 高的利用实测数据同时作为训练样本与测试样本的 通行做法对比, 误差较小, 充分验证了机械传动系 统故障诊断的个性化诊断原理具有普适性, 能解决 故障诊断过程中故障特征信息缺乏的短板，从而激 活任意类型的人工智能诊断方法。 


\section{5 结论}

（1）基于机械零部件个体差异的精准诊断需 求, 提出机械传动系统个性化故障诊断基本原理, 通过构建机械传动系统数值模型, 进行仿真分析, 获得故障样本, 作为 $\mathrm{AI}$ 模型的训练样本, 解决故障 诊断过程中因机器运行个性化特征导致的零故障样 本的短板，从而激活人工智能诊断方法。

(2) 基于有限元模拟, 具体提出有限元模拟驱 动 SVM、ELM、CNN 的轴承、齿轮、轴承一转子 系统故障个性化精准诊断系列方法。实验结果表明: 在零故障样本的条件下, 可用数值模拟方法获得故 障样本, 作为 $\mathrm{AI}$ 模型的训练样本, 获得较好的诊断 精度, 可解决实际机械传动系统故障诊断中运用 $\mathrm{AI}$ 模型时缺乏故障样本的根本性难题, 具有重要的学 术意义和应用价值。

(3) 数值模型修正, 获得具有一定精度的仿真 模型是确保诊断准确度的基础, 但是研究表明, 保 证仿真信号与实测信号具有一定的相似度的前提 下，可获得比较满意结果。而任意选取的 SVM、 ELM、CN 三种 AI 模型故障分类实验结果表明: 所 提出机械传动系统故障诊断的个性化诊断原理, 具 有一定的普适性与可拓展性。

基于机械传动系统个性化故障诊断基本原理, 进一步研究途径: (1) 分类精度进一步提高: 可结合 其他数值模拟方法(如集中参数模型方法) 实现快速 求解、更精细的数值模型修正方法实现匹配程度更 高的仿真信号、生成式对抗网络或迁移学习弥补仿 真信号与实测信号的误差导致的故障样本偏差、信 号消噪方法提纯原始信号等。(2) 变工况: 按实际工 况进行仿真, 用时频分析方法将实测信号与仿真信 号分别分解至子频段, 再采用本文原理开展故障分 类; 迁移学习或深度迁移学习亦可部分弥补实测信 号和仿真信号来源于不同工况所产生的差异。(3) 复 杂机械装备应用: 利用故障检测与隔离技术, 建立 基于状态方程的机电装备故障检测与隔离数学模 型, 实现考虑易损机械系统、电气系统耦合影响的 机电系统混合模型, 继而开展数值仿真, 实现仿真 数据与实测数据互补。

\section{参 考 文 献}

[1] 何正嘉, 陈雪峰, 李兵, 等. 小波有限元理论及工程应 用[M]. 北京: 科学出版社, 2006

HE Zhengjia, CHEN Xuefeng, LI Bing, et al. Wavelet finite element theory and engineering application[M].
Beijing: Science Press, 2006.

[2] LEI Y G. Intelligent fault diagnosis and remaining useful life prediction of rotating machinery[M]. Oxford: Elsevier Butterworth-Heinemann, 2016.

[3] HAO Y, SONG L, REN B, et al. Step-by-step compound faults diagnosis method for equipment based on majorization-minimization and constraint SCA[J]. IEEE/ASME Transactions on Mechatronics, 2019, 24(4): 2477-2487.

[4] 孙文珺, 邵思羽, 严如强. 基于稀疏自动编码深度神经 网络的感应电动机故障诊断[J]. 机械工程学报, 2016, 52(9): 65-71.

SUN Wenjun, SHAO Siyu, YAN Ruqiang. Induction motor fault diagnosis based on deep neural network of sparse auto-encoder[J]. Journal of Mechanical Engineering, 2016, 52(9): 65-71.

[5] YANG B, LEI Y G, JIA F, et al. An intelligent fault diagnosis approach based on transfer learning from laboratory bearings to locomotive bearings[J]. Mechanical Systems and Signal Processing, 2019, 122: 692-706.

[6] LEI Y G, YANG B, JIANG X W, et al. Applications of machine learning to machine fault diagnosis: A review and roadmap[J]. Mechanical Systems and Signal Processing, 2020, 138: 106587.

[7] ZHAO R, YAN R, CHEN Z, et al. Deep learning and its applications to machine health monitoring[J]. Mechanical Systems and Signal Processing, 2019, 115: 213-237.

[8] GAO Y, LIU X Y, XIANG J W. FEM simulation- based generative adversarial networks to detect bearing faults[J]. IEEE Transactions on Industrial Informatics，2020，16: 4961-4971.

[9] 钟秉林, 黄仁. 机械传动系统故障诊断学 [M]. 3 版. 北 京: 机械工业出版社, 2007.

ZHONG Binglin, HUANG Ren. Mechanical fault diagnostics[M]. 3rd ed. Beijing: China Machine Press, 2007.

[10] PERKEL J M. Molecular diagnostics : personalizing personalized medicine[J]. Science, 2009, 324(5928): 815-817.

[11] CHEN J, RANDALL R, FENG N S. Modelling and diagnosis of big-end bearing knock fault in internal combustion engines[J]. Proceedings of the Institution of Mechanical Engineers Part C-Journal of Mechanical Engineering Science, 2014, 228(16): 2973-2984.

[12] FAN L, WANG S P, WANG X J, et al. Nonlinear dynamic modeling of a helicopter planetary gear train for carrier plate crack fault diagnosis[J]. Chinese Journal of Aeronautics, 2016, 29(3): 675-687.

[13] 雷亚国, 罗希, 刘宗尧, 等. 行星轮系动力学新模型及 其故障响应特性研究[J]. 机械工程学报, 2016, 52(13): 


\section{1-122.}

LEI Yaguo, LUO Xi, LIU Zhongyao et al. A new dynamic model of planetary gear sets and research on fault response characteristics[J]. Journal of Mechanical Engineering, 2016, 52(13): 111-122.

[14] KAN M S, TAN A C C, MATHEW J. A review on prognostic techniques for non-stationary and non-linear rotating systems[J]. Mechanical Systems and Signal Processing, 2015, 62-63: 1-20.

[15] WEI S, HAN Q K, DONG X J, et al. Dynamic response of a single-mesh gear system with periodic mesh stiffness and backlash nonlinearity under uncertainty[J]. Nonlinear Dynamics, 2017, 89(1): 49-60.

[16] HAN Q K, LI, X L, CHU F L. Skidding behavior of cylindrical roller bearings under time-variable load conditions[J]. International Journal of Mechanical Sciences, 2018, 135: 203-214.

[17] ARORA V, SINGH S P, KUNDRA T K. Damped FE model updating using complex updating parameters its use for dynamic design[J]. Journal of Sound and Vibration, 2009, 324(1-2): 350-364.

[18] XIANG J W, ZHONG Y T. A novel personalized diagnosis methodology to detect faults in a shaft[J]. Applied Sciences- Basel, 2016，6(12), 414.

[19] LIU X Y, HUANG H Z, XIANG J W. A personalized diagnosis method to detect faults in gears using numerical simulation and extreme learning machine[J]. Knowledge-Based Systems， 2020， 195: 105653.

[20] LIU X Y, HUANG H Z, XIANG J W. A personalized diagnosis method to detect faults in a bearing based on acceleration sensors and a FEM simulation driving support vector machine[J]. Sensors，2020，20: 420.

[21] YANG B, SHIM J. Practical datasets for similarity measures and their threshold values[J]. Journal of Korea Game Society, 2013, 18: 97-105.

[22] LU Y, TU Z G. A two-level neural network approach for dynamic FE model updating including damping [J]. Journal of Sound and Vibration, 2004, 275(3-5): 931-952.

[23] SARIN H, KOKKOLARAS M, HULBERT G, et al. Comparing time histories for validation of simulation models : error measures and metrics[J]. Journal of Dynamic Systems Measurement and Control-Transactions of the ASME，2010， 132(6): 173-179.

[24] Case Western Reserve University. Bearing data center [EB/OL]. [2020-01-01].http : //csegroups.case.edu/ bearingdatacenter/home.

[25] ZHAO C, YU X, HUANG Q, et al. Analysis on the load characteristics and coefficient of friction of angular contact ball bearing at high speed[J]. Tribology International, 2015, 87: 50-56.

[26] HAMROCK B J, DOWSON D. Ball bearing lubrication: The elastohydrodynamics of elliptical contacts[M]. New York: John Wiley \& Sons Press, 1981.

[27] CHEN S Y, KUNG C, LIAO T T. Dynamic analysis of a rotary hollow shaft with hot-fit part using contact elements with friction[J]. Transactions of the Canadian Society for Mechanical, 2011, 35: 461-474.

[28] CHEN B, LI S S, CHEN J, et al. The sources of damping in rolling element bearings under oscillating conditions $[\mathrm{J}]$. Lubrication Engineering, 2013， 38: 62-66.

[29] HARRIS T A. Rolling bearing analysis[M]. London: John Wiley and Sons Press, 1991.

[30] VENNER C H, WIJNANT Y H. Validation of EHL contact predictions under time varying load[J]. Proceedings of the Institution of Mechanical Engineers, Part J-Journal of Engineering Tribology, 2005, 219 (4): $1-13$.

[31] DIETL P, WENSING J, NIJEN G C V, Rolling bearing damping for dynamic analysis of multi-body systemsexperimental and theoretical results[J]. Proceedings of the Institution of Mechanical Engineers, Part K-Journal of Multi-body Dynamics, 2000, 214(1): 33-43.

[32] HARRIS T A, KOTZALAS M N. Advanced concepts of bearing technology: Rolling bearing analysis[M]. New York: Taylor \& Francis Group-CRC Press, 2006.

[33] CAO H R, NIU L K, HE Z J. Method for vibration response simulation and sensor placement optimization of a machine tool spindle system with a bearing $\operatorname{defect}[\mathrm{J}]$. Sensors, 2012, 12(7): 8732-8754.

作者简介：向家伟(通信作者), 男, 1974 年出生, 博士, 教授, 博士研 究生导师。主要研究方向为机电液系统状态监测与故障诊断, 有限元/ 边界元分析, 机械动力学。

E-mail:wxw8627@163.com 